\title{
Prevalencia del bullying en México: un meta-análisis del bullying tradicional y cyberbullying*
}

\author{
Bullying prevalence in Mexico: a meta-analysis \\ of traditional bullying and cyberbullying
}

\author{
Julio Isaac Vega-Cauich ${ }^{* *}$ \\ ORCID 0000-0001-9190-3720 \\ Coordinación de Investigación \\ y Medición Psicológica \\ Foco Rojo, Centro de Psicología \\ Aplicada, México
}

Recibido: 11 de enero de 2018 Revisado: 26 de febrero de 2018 Aceptado: 4 de mayo de 2018

\section{Resumen}

Objetivo: Sintetizar los diagnósticos de bullying en México para estimar la prevalencia de víctimas y agresores, tanto en su modalidad cara a cara como virtual. Método: Se realizó un meta-análisis de la prevalencia con 27 investigaciones realizadas en México con un modelo de efectos aleatorios, y se exploraron sus moderadores a través de una meta-regresión. Resultado: La prevalencia osciló entre el 19 y $21 \%$ para cibervictimización, victimización y agresión cara a cara, y de un $11 \%$ para la ciberagresión. Las variables que moderaron la estimación de la prevalencia fueron la presencia de una definición en el instrumento y el número de ítems. Ningún estudio utilizó instrumentos que evaluaran el componente de desbalance de poder del bullying, por lo que es probable que los estudios hayan evaluado el concepto de victimización entre pares. Conclusión: Uno de cada cinco estudiantes mexicanos recibe o realiza bullying o cyberbyllying. Se proponen recomendaciones metodológicas para futuras investigaciones.

Palabras clave: meta-análisis, revisión sistemática, acoso escolar, bullying, prevalencia.

Artículo de investigación. Citar como: Vega-Cauich, J. I. (2019). Prevalencia del bullying en México: un meta-análisis del bullying tradicional y cyberbullying. Diversitas: Perspectivas en Psicología, 15(1), 111-127. DOI: https://doi.org/10.15332/s1794-9998.2019.0001.09

Correspondencia: Julio Isaac Vega-Cauich, Coordinación de Investigación y Medición Psicológica, Foco Rojo, México. Dirección postal: Calle 47 No. 506 por 62 y 64 Centro, Mérida, Yucatán, México. C.P. 97000. Correo electrónico: julio.vega@outlook.com 


\section{Abstract}

Objective: Summarize the bullying studies in Mexico to estimate the prevalence for both victims and aggressors, both face-to-face and cyberbullying. Method: A meta-analysis of the prevalence was carried out with 27 investigations carried out in Mexico with a random effects model, and its moderators were explored through a meta-regression. Result: The prevalence ranges were from $19 \%$ to $21 \%$ for cyber-victimization, face-to-face victimization and face-to-face aggression, and $11 \%$ for cyberaggression. The variables that moderated the prevalence estimation were the presence of a definition in the instrument and the number of items. Neither study uses tools that evaluate the power imbalance component of bullying, so it is likely that studies evaluated the concept of peer victimization. Conclusion: One in five Mexican students receives or performs bullying or cyberbyllying. Methodological recommendations are proposed for future research.

Keywords: meta-analysis, review, bullying, prevalence.

\section{Introducción}

El acoso escolar o bullying en México es un problema actual. Algunos reportes sugieren que el país ocupa los primeros lugares en cuanto al bullying a nivel mundial (Valadez, 2014). Además, los estudios han demostrado que existe una asociación entre el bullying y los problemas de salud mental que van desde la depresión hasta el suicidio (Bauman, Toomey, \& Walker, 2013; Gini \& Pozzoli, 2009). Estas consecuencias han motivado que la investigación en años recientes vaya aumentando de forma consistente (Dibomicucci Brazil, Basso Meneghini, de Souza Costa, \& Lemes, 2015). Sin embargo, uno de los principales retos que tiene el estudio del bullying es un adecuado diagnóstico, pues sin una correcta medición no se puede asegurar un adecuado plan de intervención (Crothers \& Levinson, 2004).

Los estudios de prevalencia del bullying han sido variados, sin embargo, recientemente destaca el trabajo de Modecki, Minchin, Harbaugh, Guerra y Runions (2014), donde sistematizan 80 estudios sobre el bullying y cyberbullying a nivel mundial para realizar un meta-análisis de su prevalencia. Modecki y su equipo analizaron la prevalencia del bullying tradicional (cara a cara) y el cyberbullying, tanto para víctimas como para agresores. En este mismo trabajo, también se analizaron variables que podrían moderar la estimación de la prevalencia, como por ejemplo, si el instrumento proveía una definición o el uso de ejemplos, si utilizó palabras sensibles (como usar bully en los reactivos), o si la muestra fue aleatoria. Los resultados señalaron que, tanto para víctimas como para agresores, la prevalencia del bullying tradicional fue mayor que la del cyberbullying, aproximadamente de un 35\% y $15 \%$ respectivamente. Además, el estudio determinó que estas prevalencias se encontraban correlacionadas, lo que sugiere que el cyberbullying, con una menor prevalencia que el bullying, es un tipo de expresión de éste, y no un fenómeno independiente. El estudio además señala la importancia de algunos aspectos metodológicos que podrían moderar la prevalencia estimada. Por una parte, brindar una definición clara que incluye los tres elementos que componen el bullying aumenta la estimación de la prevalencia. Por el contrario, brindar ejemplos, utilizar la palabra "bullying" o utilizar un muestreo probabilístico, disminuye la estimación de la prevalencia. Estas conclusiones ya habían sido señaladas por estudios previos (Felix, Sharkey, Green, Furlong, \& Tanigawa, 2011; Green, Felix, Sharkey, Furlong, \& Kras, 2013). Sin embargo, otras variables como el tipo y diseño del muestreo han sido poco abordados previamente.

Otro de los problemas que continuamente se menciona es el tipo de instrumento, debido a que la variedad de instrumentos utilizados también puede 
afectar la estimación de la prevalencia (Ybarra, Espelage, \& Mitchell, 2014). Esto se debe principalmente a que los instrumentos utilizados no siempre evalúan todos los elementos que componen el bullying, que corresponden a (1) la intencionalidad de hacer daño, (2) en un periodo prolongado de tiempo, (3) y la existencia de desbalance de poder (Vivolo-Kantor, Martell, Holland, \& Westby, 2014). Este último, un componente esencial para diferenciar el bullying de otras formas de violencia escolar (Ybarra et al., 2014).

A nivel Iberoamérica también destaca el trabajo realizado por Zych, Ortega-Ruiz \& Marín-López (2016), donde sintetizan los estudios de acoso escolar realizados en España de 2008 a 2015 sobre cyberbullying. En la revisión sistemática realizada, los autores concluyen sobre la gran influencia que puede tener el diseño del estudio y la instrumentación utilizada (por ejemplo, si el instrumento utilizó un solo ítem o varios para evaluar el ciberbullying). De igual forma, reportan el promedio de la prevalencia para diferentes formas de involucramiento en el cyberbullying. En los estudios que evaluaron el cyberbullying a través de diferentes escalas con varios ítems, el promedio para víctimas y agresores fue bastante similar con una prevalencia promedio de $24 \%$ y $26 \%$ respetivamente. Cuando esta estimación se realizaba con un solo ítem, la prevalencia disminuyó a un $10 \%$ y $14 \%$ respectivamente. Sin embargo, cabe recalcar que el estudio realizado utilizó el promedio simple de las prevalencias, una práctica no recomendada para sintetizar resultados de diversos estudios debido a que la calidad de los estudios no necesariamente es la misma. Por lo que se recomiendan técnicas de ponderación como el meta-análisis para realizar la síntesis del efecto global (Borenstein, Hedges, Higgins, \& Rothstein, 2009). Finalmente, hay que recalcar que el estudio no reportó el manejo del sesgo en las publicaciones, por lo que los resultados también deben ser analizados con reservas al generalizar solo a estudios publicados en revistas arbitradas.

En México, los estudios que sinteticen los principales hallazgos sobre bullying son escasos. Valdés Cuervo y sus colaboradores (2016) analizaron 53 estudios realizados en México de 2001-2014. Sin embargo, el estudio es solo un análisis bibliométrico de los estudios de bullying en México, y no integra o sintetiza de ninguna forma los resultados y sus metodologías. Esta falta de síntesis de hallazgos motiva la presente investigación. El propósito entonces fue sintetizar, mediante un meta-análisis, las prevalencias de bullying en los estudios realizados en México. El estudio se basó en la metodología presentada por Modecki et al. (2014) con las adecuaciones correspondientes para integrar los resultados de los estudios realizados en México.

\section{Método}

\section{Selección de los estudios}

Criterios de elegibilidad: Se consideraron únicamente artículos que abordaran el tema de bullying realizados en México. Para esto, se consideraron los siguientes criterios de inclusión: 1) haber sido realizado con muestra y participantes de México; 2 ) que abordara el tema de bullying y/o cyberbullying; 3) que brindara información que permitiera estimar la prevalencia (tamaño de la muestra y cantidad de alumnos que realizan o reciben bullying). Los estudios realizados por autores o instituciones mexicanas fueron incluidos, para posteriormente verificar el cumplimiento del primer criterio de inclusión. Se eliminaron todos aquellos estudios que abordaron el bullying laboral, o que fueran estudios de tipo narrativo/teórico. No se fijó una fecha de inicio, pues se intentó abarcar la mayor cantidad de estudios posibles en México. Sin embargo, se consideró una fecha límite para no incluir estudios posteriores que pudieran ser publicados durante la elaboración de este documento, fijando como fecha límite el 1 de marzo de 2017.

Estrategia de búsqueda: Se utilizaron las bases de datos de Scopus, Web of Science, Ebsco, Scielo, Redalyc, así como la búsqueda manual en los perfiles de ResearchGate de los autores más frecuentes, y la revisión de las memorias de investigación del Congreso Mexicano de Investigación Educativa, con el objetivo de evitar el sesgo en la publicación. Para la búsqueda de artículos se utilizaron los términos "bullying", "acoso escolar", "hostigamiento escolar", "cyberbullying” y "maltrato entre pares". El resultado del proceso de búsqueda dio la inclusión a 27 estudios (ver figura 1). 
Figura 1. Diagrama de flujo de la selección de artículos.

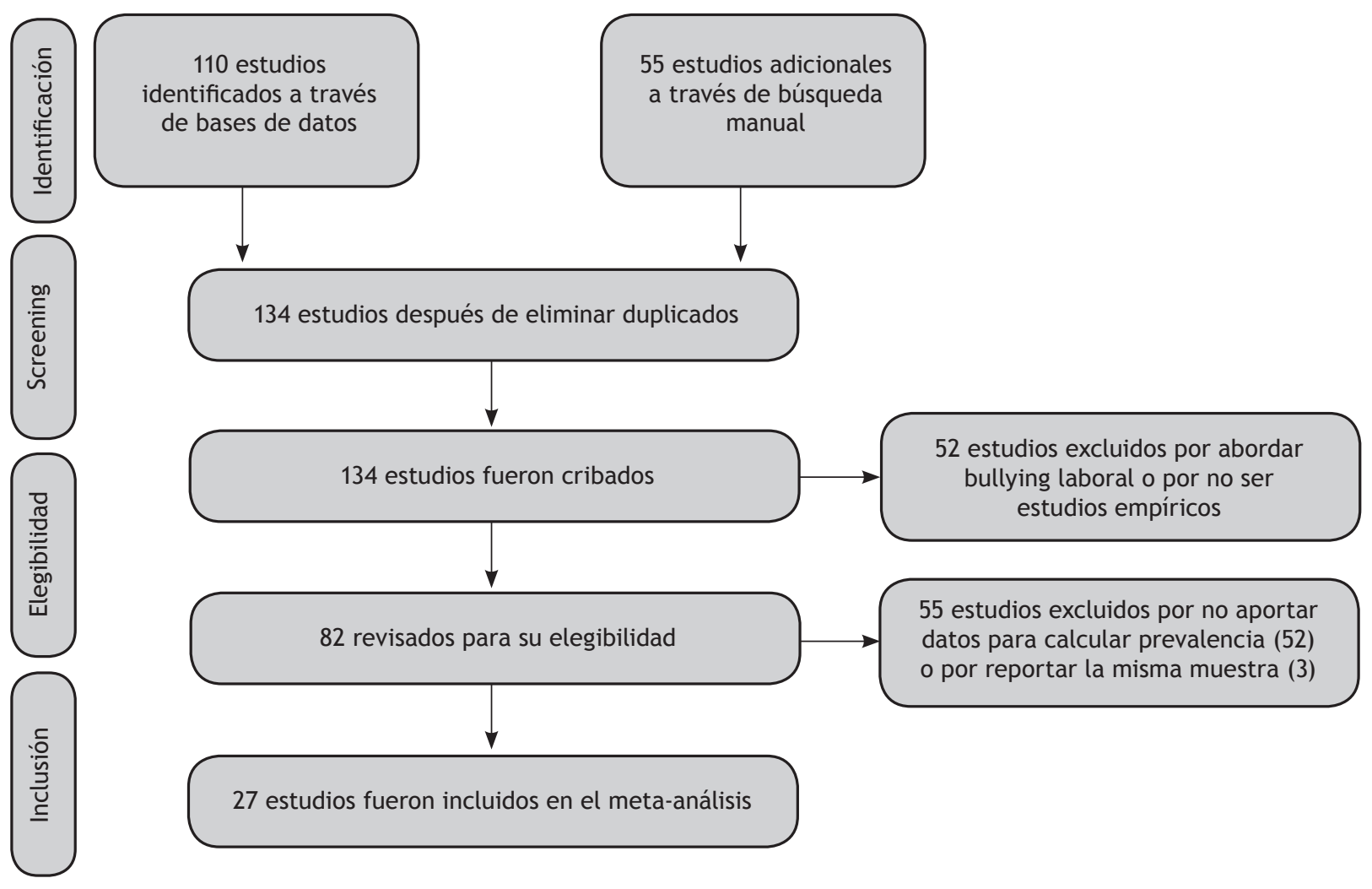

\section{Codificación de la información}

A partir de los artículos hallados, se consideró recoger la siguiente información: el constructo evaluado (bullying o cyberbullying), el tipo de muestreo utilizado; el tipo de instrumentación elegido y sus características (uso de una definición u operacionalización de diferentes tipos de agresión; el uso o no de términos sensibles como bully o acoso en los ítems o definición; y el número de ítems para evaluar el constructo), así como el tamaño de muestra y la prevalencia de bullying (tabla 1). Los estudios fueron revisados por dos evaluadores independientes, y todas las dudas o conflictos sobre su codificación fueron resueltas a través de un tercer revisor, todo esto siguiendo las recomendaciones y lineamientos para realizar estudios meta-analíticos de prevalencia (The Joanna Briggs Institute, 2014). 


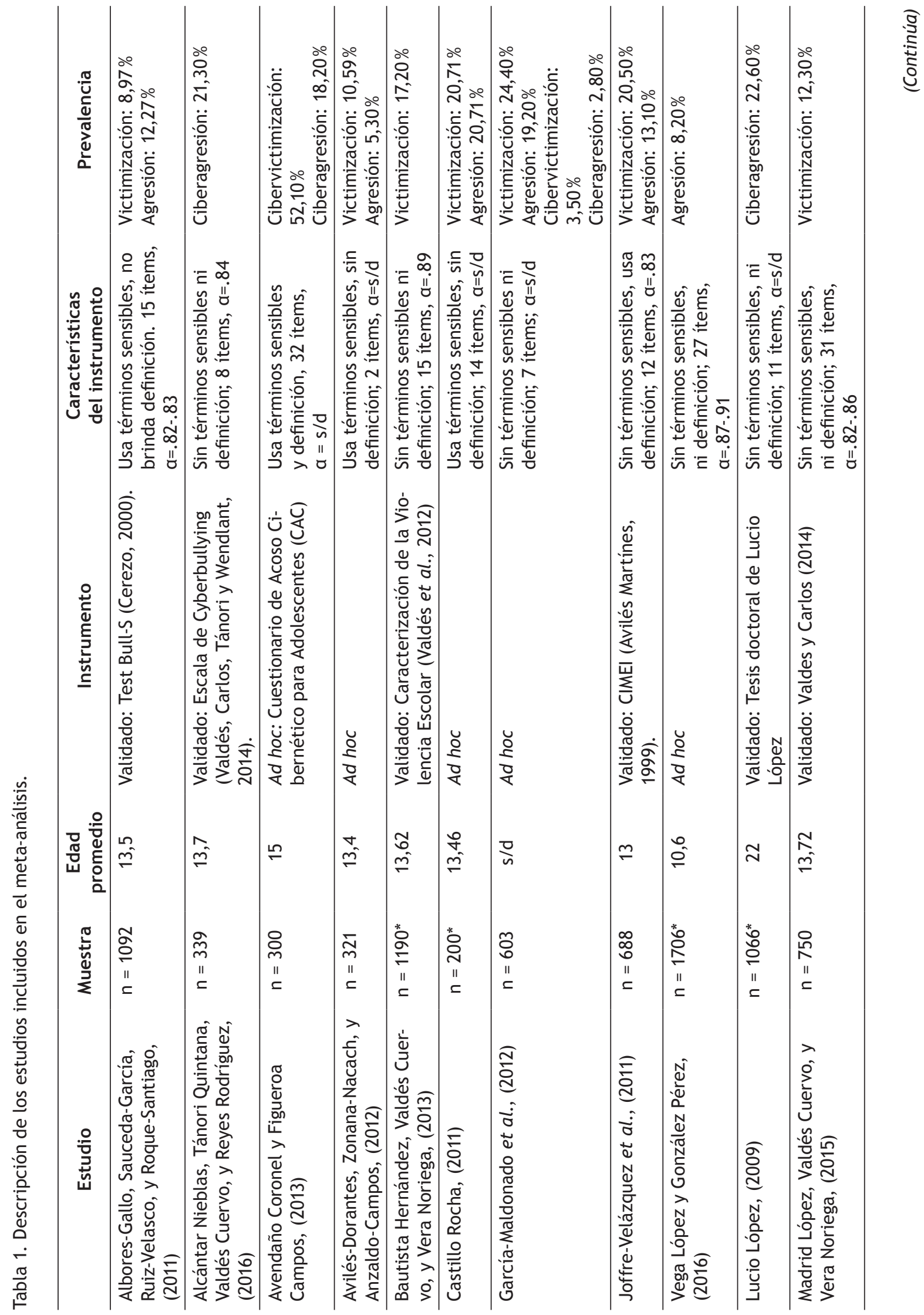




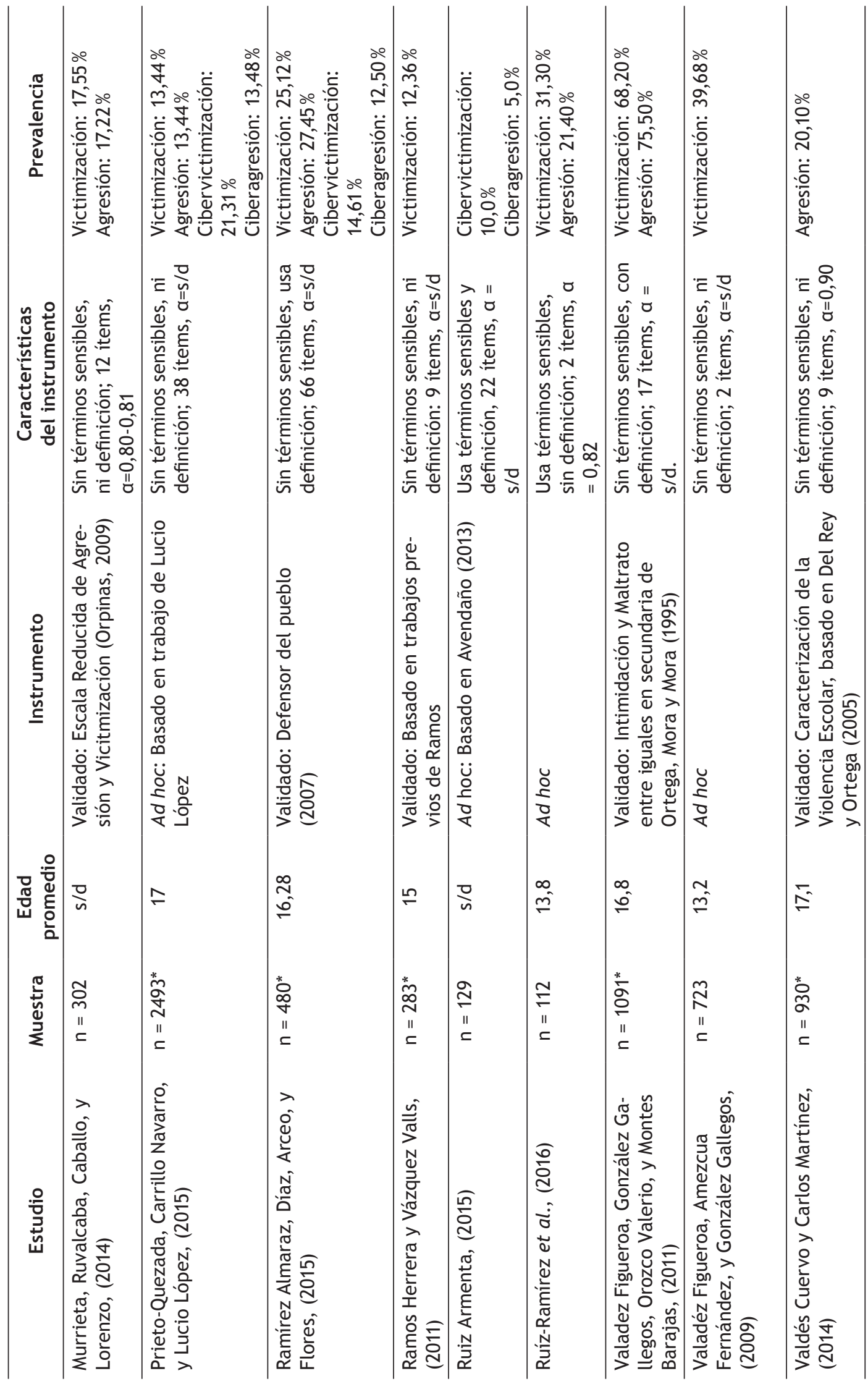




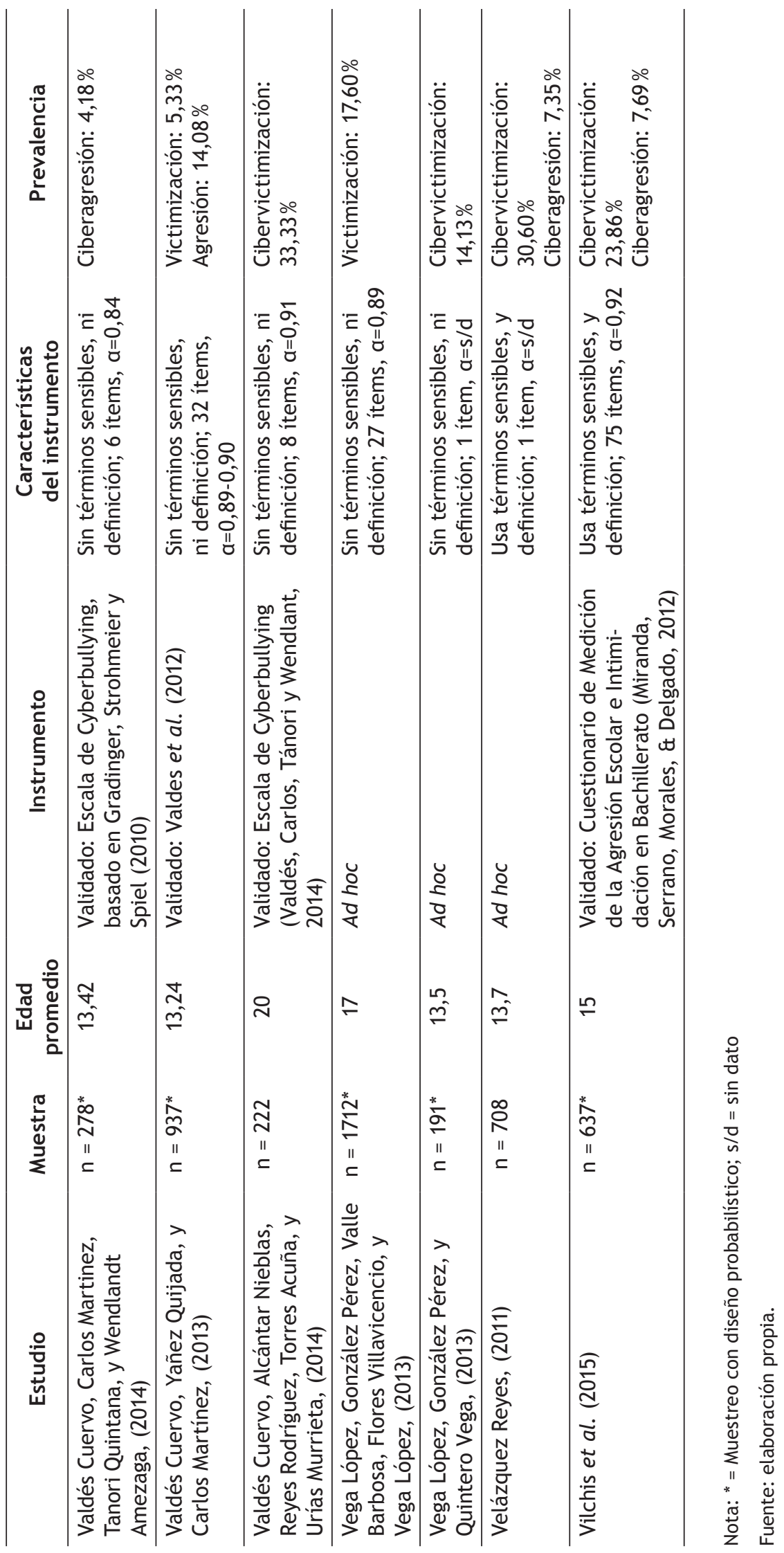




\section{Análisis de la información}

Se recogieron datos sobre la prevalencia, ya sea por víctimas, agresores o ambos, así como por su modalidad (acoso cara a cara o ciberacoso). Aquellos reportes que brindaban más de una modalidad de agresión fueron promediados para obtener una solo. Solo dos reportes brindaban un estimado general (sin especificar si era de víctimas o agresores), los cuales fueron utilizados en ambas categorías para los cálculos. Finalmente, para aquellos reportes que utilizaron la misma muestra para reportar distintos análisis (Valdés Cuervo, Martínez \& Torres Acuña, 2012; Valdés Cuervo, Ojeda García, Urías Murrieta \& Ponce Segura, 2013; Valdés Cuervo, Sanchez Escobedo \& Martínez, 2012), solo se consideró un reporte, tomando en cuenta aquel reporte que haya sido publicado en una revista con revisión por pares, y en caso de haber más de uno, se tomó como referencia aquel con la fecha más reciente.

Para realizar los análisis se consideró primero una transformación a los datos de prevalencia a partir de la transformación de doble arcoseno de FreemanTukey, recomendada para realizar meta-análisis de proporciones (Barendregt, Doi, Lee, Norman, \& Vos, 2013). Los datos fueron analizados con el programa estadístico $R$ (R Core Team, 2017) utilizando el paquete estadístico "meta" (Schwarzer, 2007). Para los análisis se consideró un modelo de efectos aleatorios debido a la variación en los estudios y sus metodologías (los artículos utilizaron diferentes instrumentos, niveles educativos e incluso diseños muestrales), además de que la variabilidad de éstos fueron corroborados con los correspondientes diagnósticos de heterogeneidad, demostrando que existían variaciones importantes entre los estudios (Borenstein et al., 2009). Finalmente, también se analizaron los instrumentos utilizados, especialmente con referencia al constructo evaluado y su correspondencia con los componentes de la definición del bullying. Lo anterior se realizó a partir del rastreo de la descripción de los instrumentos utilizados en la sección del método de los artículos analizados, o bien a través de la recuperación de los estudios de validación original en casos en donde habían utilizado instrumentos previamente validados.

\section{Resultados}

\section{Descripción de los estudios}

De los 27 estudios considerados en esta revisión, el $70,4 \%$ de ellos estudió el bullying cara a cara, y un $44,4 \%$ el cyberbullying. Un 7,4\% fueron realizados con participantes de nivel primaria, $48,1 \%$ secundaria; $37 \%$ bachillerato; y un $11,1 \%$ a nivel superior. De igual forma con respecto al diseño metodológico, se obtuvo que un $44,4 \%$ de los estudios utilizó un diseño de muestreo probabilístico y 55,6\% utilizó un instrumento previamente validado. El promedio de ítems utilizados en los estudios es de 18,55. Sin embargo, dado algunos valores extremos se consideró que la mediana de ítems reflejaba de mejor forma la cantidad de ítems utilizada por la mayoría de los instrumentos, la cual correspondió a 12 ítems.

Cabe recordar que la búsqueda no solo incluyó artículos de revistas arbitradas (63\%), sino también reportes de conferencias (33,3\%), y libros $(3,7 \%)$; con la intención de evitar el sesgo de publicación. Lo anterior fue logrado exitosamente, pues al explorar el sesgo en la publicación por medio de la correlación de rangos entre el error estándar y el efecto estimado en cada modelo, también conocido como prueba de Begg y Mazumdar (Schwarzer, 2007), el resultado fue que todos los estadísticos tuvieron un valor estandarizado menor a $z=1,96$, por lo que se concluyó la ausencia de sesgo en las publicaciones tanto para la victimización cara-a-cara $(z=1,17, p=0,242)$, como para la agresión cara-a-cara $(z=1,58, p=0,113)$, la cibervictimización $(z=-0,42, p=0,677)$, y la ciberagresión $(z=-0,45, p=0,655)$.

\section{Prevalencia del bullying cara a cara y cyberbullying}

En la Figura 2 se puede apreciar la síntesis de la prevalencia del bullying cara a cara, tanto para víctimas (victimización, con una muestra combinada de $\mathrm{n}=$ 12,977) como para agresores (agresión; muestra combinada de $n=10,955)$. Las prevalencias son similares, con una proporción del 20\% (I.C. 95\% $=13 \%-28 \%$ ) para la victimización, y de 19\% (I.C. 95\% = 11\%-30\%) para la agresión. Con respecto al bullying por medios electrónicos, la cibervictimización (con una muestra 
combinada de $n=5,763$ ) tuvo una prevalencia similar a la encontrada en la victimización tradicional, con un $21 \%$ (I.C. $95 \%=13 \%-30 \%$ ). Sin embargo, la ciberagresión (con una muestra combinada de $n=7,033$ ) tuvo una prevalencia menor, con un $11 \%$ (I.C. $95 \%$ $=7 \%-15 \%$ ) en su estimación. Finalmente, tal como puede observarse en la Figura 2 debajo del análisis de cada subgrupo, existe una gran variabilidad (heterogeneidad) entre los estudios $\left(I^{2}=0,97-99\right.$ que corresponde del 97 al $99 \%$ ), siendo la menor de un $97 \%$, por lo que resultó apropiado un análisis de moderadores de la prevalencia.

Figura 2. Prevalencia del acoso escolar en cuatro formas de expresión.

\section{A) Victimización}

Estudio

Albores-Gallo et al. (2011)

Aviles-Dorontes et al. (2012)

Bautista et al. (2013)

Castillo Rocha (2011)

Garcia-Maldonado et al. (2012)

Joffre-Velazquez et al. (2011)

Madrid Lopez et al. (2015)

Murrieta et al. (2014)

Prieto Quezada et al. (2015)

Ramirez Almaraz et al. (2015)

Ramos y Vazquez (2011)

Ruiz-Ramirez et al. (2016)

Valadez Figueroa et al. (2011)

Valadez Figueroa et al. (2009)

Valdes Cuervo et al. (2013)

Vega Lopez et al. (2013)

Modelo de efectos aleatorios

Heterogeneity: $I^{2}=99 \%, \tau^{2}=0.037, p<0.01$

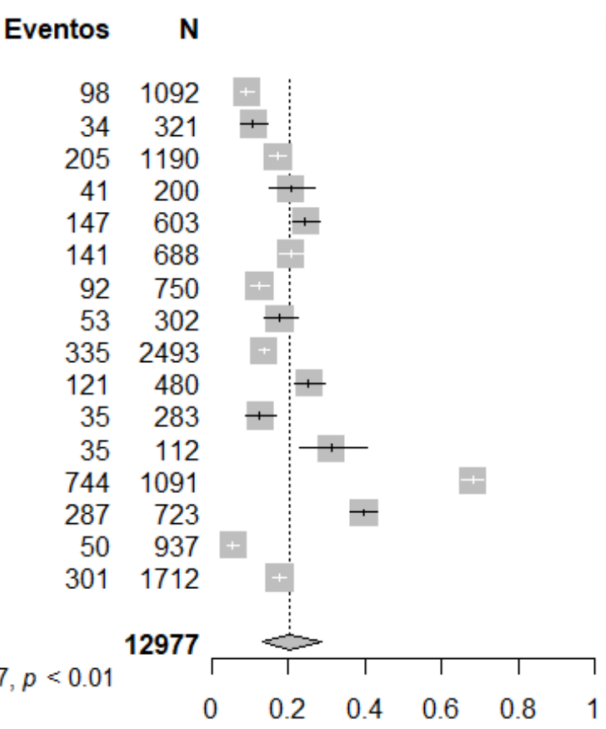

\section{B) Agresión}

Estudio

Albores-Gallo et al. (2011)

Aviles-Dorontes et al. (2012)

Castillo Rocha (2011)

Garcia-Maldonado (2012)

Joffre-Velazque et al. (2011)

Vega-Lopez y Perez (2016)

Murrieta (2014)

Prieto Quezada et al. (2015)

Ramirez Almaraz et al. (2015)

Ruiz-Ramirez et al. (2016)

Valadez Figueroa et al. (2011)

Valdes y Carlos (2014)

Valdes Cuervo et al. (2013)

Modelo de efectos aleatorios

Heterogeneity: $I^{2}=99 \%, \tau^{2}=0.0507, p<0.01$

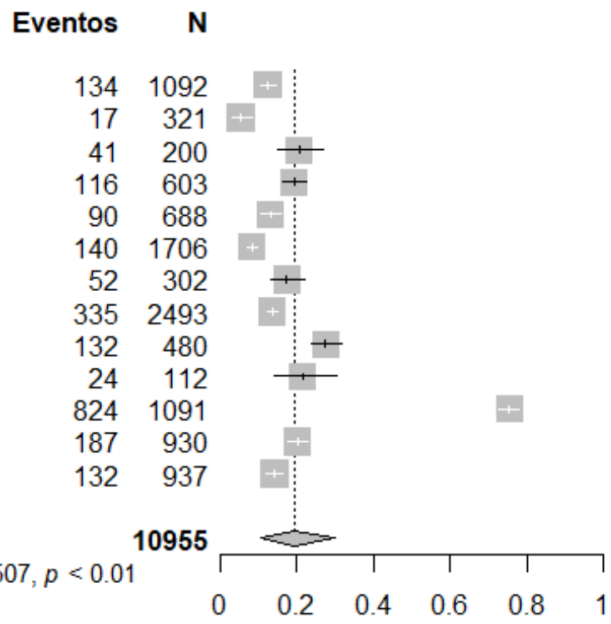

Prevalencia Int.Conf. 95\% Peso aleatorio

$\begin{array}{ll}0.09 & {[0.07 ; 0.11]} \\ 0.11 & {[0.07 ; 0.14]} \\ 0.17 & {[0.15 ; 0.19]} \\ 0.20 & {[0.15 ; 0.27]} \\ 0.24 & {[0.21 ; 0.28]} \\ 0.20 & {[0.18 ; 0.24]} \\ 0.12 & {[0.10 ; 0.15]} \\ 0.18 & {[0.13 ; 0.22]} \\ 0.13 & {[0.12 ; 0.15]} \\ 0.25 & {[0.21 ; 0.29]} \\ 0.12 & {[0.09 ; 0.17]} \\ 0.31 & {[0.23 ; 0.41]} \\ 0.68 & {[0.65 ; 0.71]} \\ 0.40 & {[0.36 ; 0.43]} \\ 0.05 & {[0.04 ; 0.07]} \\ 0.18 & {[0.16 ; 0.19]}\end{array}$

$6.3 \%$

$6.2 \%$

$6.3 \%$

$6.1 \%$

$6.3 \%$

$6.3 \%$

$6.3 \%$

$6.2 \%$

$6.3 \%$

$6.3 \%$

$6.2 \%$

$6.0 \%$

$6.3 \%$

$6.3 \%$

$6.3 \%$

$6.3 \%$

$0.20 \quad[0.13 ; 0.28] \quad 100.0 \%$
Prevalencia Int.Conf. 95\% Peso aleatorio

$\begin{array}{lll}0.12 & {[0.10 ; 0.14]} & 7.7 \% \\ 0.05 & {[0.03 ; 0.08]} & 7.7 \% \\ 0.20 & {[0.15 ; 0.27]} & 7.6 \% \\ 0.19 & {[0.16 ; 0.23]} & 7.7 \% \\ 0.13 & {[0.11 ; 0.16]} & 7.7 \% \\ 0.08 & {[0.07 ; 0.10]} & 7.8 \% \\ 0.17 & {[0.13 ; 0.22]} & 7.7 \% \\ 0.13 & {[0.12 ; 0.15]} & 7.8 \% \\ 0.28 & {[0.24 ; 0.32]} & 7.7 \% \\ 0.21 & {[0.14 ; 0.30]} & 7.5 \% \\ 0.76 & {[0.73 ; 0.78]} & 7.7 \% \\ 0.20 & {[0.18 ; 0.23]} & 7.7 \% \\ 0.14 & {[0.12 ; 0.16]} & 7.7 \%\end{array}$

$0.19[0.11 ; 0.30] \quad 100.0 \%$

(Continúa) 


\section{C) Cibervictimización}

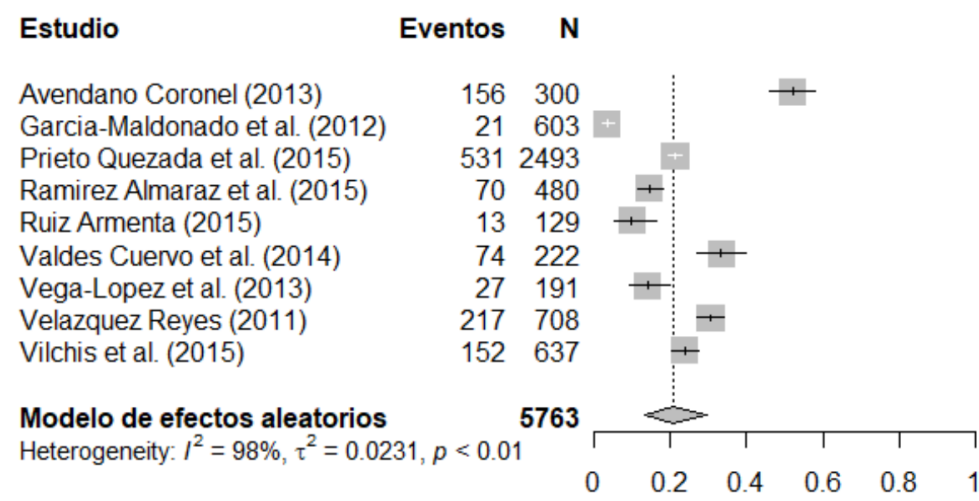

Prevalencia Int.Conf. 95\% Peso aleatorio

$\begin{array}{llr}0.52 & {[0.46 ; 0.58]} & 11.1 \% \\ 0.03 & {[0.02 ; 0.05]} & 11.3 \% \\ 0.21 & {[0.20 ; 0.23]} & 11.4 \% \\ 0.15 & {[0.12 ; 0.18]} & 11.2 \% \\ 0.10 & {[0.05 ; 0.17]} & 10.6 \% \\ 0.33 & {[0.27 ; 0.40]} & 10.9 \% \\ 0.14 & {[0.10 ; 0.20]} & 10.9 \% \\ 0.31 & {[0.27 ; 0.34]} & 11.3 \% \\ 0.24 & {[0.21 ; 0.27]} & 11.3 \% \\ & & \\ \mathbf{0 . 2 1} & {[\mathbf{0 . 1 3} ; \mathbf{0 . 3 0}]} & \mathbf{1 0 0 . 0 \%}\end{array}$

\section{D) Ciberagresión}

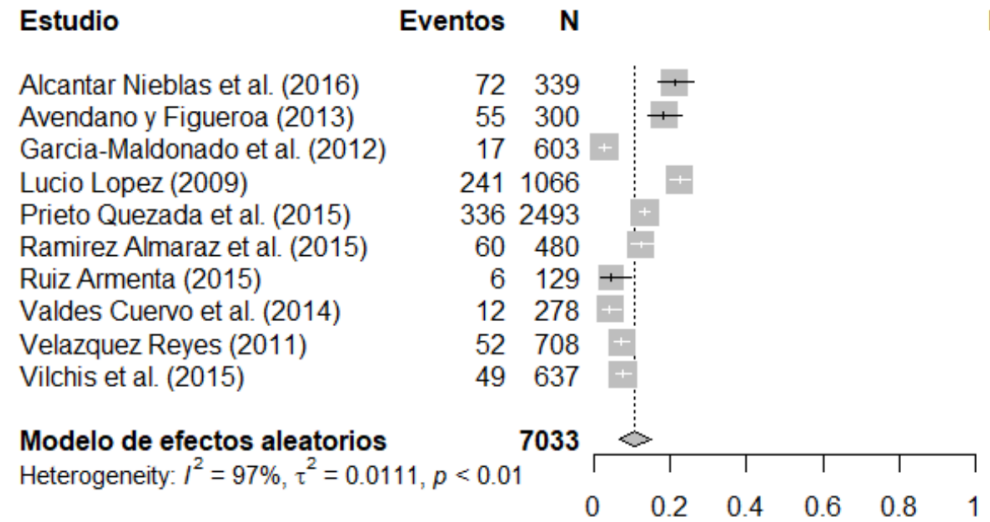

Prevalencia Int.Conf. 95\% Peso aleatorio

$\begin{array}{llr}0.21 & {[0.17 ; 0.26]} & 9.9 \% \\ 0.18 & {[0.14 ; 0.23]} & 9.8 \% \\ 0.03 & {[0.02 ; 0.04]} & 10.2 \% \\ 0.23 & {[0.20 ; 0.25]} & 10.3 \% \\ 0.13 & {[0.12 ; 0.15]} & 10.5 \% \\ 0.12 & {[0.10 ; 0.16]} & 10.1 \% \\ 0.05 & {[0.02 ; 0.10]} & 9.0 \% \\ 0.04 & {[0.02 ; 0.07]} & 9.8 \% \\ 0.07 & {[0.06 ; 0.10]} & 10.2 \% \\ 0.08 & {[0.06 ; 0.10]} & 10.2 \% \\ & & \\ \mathbf{0 . 1 1} & {[\mathbf{0 . 0 7} \mathbf{0 . 1 5}]} & \mathbf{1 0 0 . 0 \%}\end{array}$

Nota: En el diagrama de bosque (forest plot) se dividen los análisis según el tipo de prevalencia observada en cuatro grupos: A) Victimización, B) Agresión, C) Cibervictimización y D) Ciberagresión. La escala de prevalencia corresponde a una proporción que va de 0 a 1 , que en términos de porcentajes equivale del 0 a 100\%. Los resultados individuales de cada estudio están representados con un cuadrado y su correspondiente intervalo de confianza con una línea que atraviesa el cuadrado. A un mayor tamaño del cuadrado, mayor importancia del estudio (peso aleatorio). La prevalencia global que integra la información de todos los estudios de cada grupo es representada por la figura en forma de diamante (tamaño del efecto), cuyo largo corresponde al largo del intervalo de confianza de la prevalencia global. De tal forma, existe una prevalencia global para cada grupo de análisis. Adicionalmente se proporcionan otros datos como el tamaño de la muestra $(\mathrm{N})$, la cantidad de estudiantes involucrados en el acoso (Eventos), y los intervalos de confianza de la prevalencia de cada estudio (Int. Conf. 95\%). 


\section{Moderadores de la prevalencia}

Se analizaron los siguientes moderadores: forma de medición (uso de definiciones o la operacionalización de indicadores); uso o no de términos sensibles en los ítems o definición; el número de ítems utilizado por el instrumento, el tipo de muestreo realizado (probabilístico o no probabilístico) y la edad de los participantes, todo esto controlado por el subgrupo analizado. A partir de una meta-regresión, se encontró que el uso de una definición vs la operacionalización, explican de forma significativa la prevalencia $(B=0,23 ; z=4,17 ; p<0,001)$. El coeficiente obtenido señala que el uso de definiciones presentadas a los participantes aumenta significativamente la estimación de la prevalencia. También se obtuvo que el número de ítems utilizado en los instrumentos modera la prevalencia $(B=-0,0036$, $z=-2,54 ; p=0,011)$. El valor del coeficiente obtenido señala que una menor cantidad de reactivos tiende a sobreestimar la prevalencia del acoso escolar. Finalmente, ni la edad, el tipo de muestreo elegido o el uso de términos sensibles se relacionó con las prevalencias estimadas en los estudios.

\section{Análisis sobre la instrumentación utilizada}

Finalmente, también fueron analizados los instrumentos utilizados en los estudios revisados. Se encontró que un $22,2 \%(n=6)$ de los instrumentos empleó una definición del constructo de bullying o cyberbullying que se presentó a los participantes para determinar su prevalencia. De igual forma, un 29,6\% $(n=8)$ de los instrumentos utilizó términos sensibles en el instrumento o sus reactivos, tales como bully o acoso.

Los instrumentos empleados en los estudios revisados no son mayoritariamente utilizados según la revisión de Vivolo-Kantor et al. (2014), con excepción de las escalas basadas en el trabajo de Orpinas (2009) y el Test Bull-S (Cerezo, 2000). La mayoría de los instrumentos fueron construidos para investigaciones realizadas en México, o en caso de haber sido validados previamente, habían sido poco utilizados a nivel internacional. Se observó que todos los instrumentos evaluaron el componente de intención de causar un daño, y la repetición de estas conduc- tas. Sin embargo, solamente dos instrumentos evaluaron el componente de desbalance de poder: el CIMEI o Cuestionario sobre Intimidación y Maltrato Entre Iguales (Avilés Martínes, 1999) y el Test Bull-S. Pero, ninguno de ellos lo utiliza para la determinación de la presencia o ausencia del bullying. Es decir, dado de que ningún instrumento utilizado considera los tres componentes del bullying para su evaluación, no se puede considerar en los estudios revisados que hayan medido el bullying de forma estricta, sino quizás constructos relacionados como la victimización por pares. A pesar de lo anterior, la mayoría de los instrumentos reportan valores buenos de consistencia interna que iban de 0,81 hasta 0,92; lo que habla de una buena consistencia para evaluar la victimización por pares en la escuela.

\section{Discusión}

Los resultados señalan que los estudios analizados probablemente no hayan evaluado el constructo de bullying, pues los instrumentos utilizados no contemplan todos los elementos del bullying para su respectivo diagnóstico. En particular, no contemplan el componente de desbalance de poder, un componente que las investigaciones internacionales han señalado como esencial para diferenciar el acoso escolar de otras formas de agresión (Green et al., 2013; Volk, Dane \& Marini, 2014; Volk, Veenstra \& Espelage, 2017; Ybarra et al., 2014). Es muy probable que se hayan evaluado constructos como la victimización entre pares, o incluso la violencia escolar. De hecho, algunos estudios que basan sus mediciones en los instrumentos de Del Rey y Ortega (Valdés Cuervo, Martinez, Tanori Quintana \& Wendlandt Amezaga, 2014), deben considerar que las mismas autoras señalan que su instrumento no mide únicamente bullying, sino otros aspectos más amplios de la violencia escolar, específicamente la violencia interpersonal que ocurre en los centros escolares (Del Rey \& Ortega, 2005). Esto es similar a lo encontrado por Zych, Ortega-Ruiz y Marín-López (2016) que señalan que los estudios de ciberbullying pueden ser considerados como estudios de un concepto más amplio como la ciberagresión.

Es por tanto necesario enmarcar las prevalencias encontradas en el contexto de la victimización por pares y no específicamente dentro del concepto 
de bullying. A pesar de lo anterior, los estudios realizados no son menos importantes, ya que la atención a cualquier forma de agresión debe ser considerada como fundamental para el desarrollo adecuado de las personas, por tanto, aunque posiblemente los estudios evaluaron el concepto de victimización por pares, sus hallazgos siguen siendo fundamentales para el entendimiento y prevención de la violencia escolar.

Con respecto a los cálculos de la prevalencia, llama la atención que las estimaciones son menores a las encontradas por Modecki et al. (2014). Lo anterior posiblemente se deba a tres razones: 1) la gran variabilidad en los instrumentos utilizados, 2) los tipos de muestra, y 3 ) el hecho que posiblemente no se esté midiendo el bullying, sino victimización entre pares. Los resultados de este estudio también contrastan con reportes internacionales que señalan a México como uno de los países con mayor prevalencia de bullying. Es posible que la discrepancia anterior se deba al concepto y tipo de instrumento utilizado para las estimaciones de los estudios internacionales, pues la variabilidad en los instrumentos ha sido un problema comúnmente señalado por los expertos en acoso escolar que comúnmente dificulta la comparabilidad entre países (Furlong, Sharkey, Felix, Tanigawa, \& Green, 2010).

Sin embargo, hay que destacar que los intervalos de confianza obtenidos en el meta-análisis contienen las estimaciones propuestas por Modecki. Además, los resultados son más similares a los encontrados por Zych et al. (2016) en España, con reportes cercanos al $20 \%$ para varias medidas de victimización y agresión. Lo anterior quizás sea el reflejo de similitudes culturales en ambos países. Además, las estimaciones encontradas en México son de gran utilidad para la planificación de futuras investigaciones. Las estimaciones pueden ser utilizadas para generar cálculos de muestreos probabilísticos que tomen en consideración la dinámica del acoso escolar en México.

Por otra parte, también destaca que el uso de definiciones afecta los cálculos de prevalencia, al aumentar la proporción estimada cuando se presenta una definición a los participantes. Esto es similar a lo que otros estudios han señalado, en cuanto a que al brindar una definición puede aumentar las estimaciones de la prevalencia (Green et al., 2013; Modecki et al., 2014). Es por esto que se recomienda trabajar en la adaptación de instrumentos ampliamente utilizados o recomendados que permita la evaluación adecuada de la prevalencia, y al mismo tiempo la comparabilidad entre diversos países (Swearer, Siebecker, Johnsen-Frerichs, \& Wang, 2010), considerando la adaptación de instrumentos que operacionalicen el constructo en lugar de brindar una definición (Felix et al., 2011; Green et al., 2013).

Otro aspecto relevante es el efecto moderador que tiene la cantidad de ítems de los instrumentos en la estimación de la prevalencia. Contrario a lo reportado por Zych et al. (2016), este meta-análisis encontró que, al menos en México, una menor cantidad de ítems está asociado a una prevalencia mayor. Es posible que esta diferencia hallada se deba a dos factores. Por una parte, el estudio de Zych, Ortega-Ruiz y Marín-López evaluó únicamente cyberbullying, mientras que los análisis presentados también incluyen el bullying cara-a-cara. Por otra parte, la síntesis brindada por Zych, Ortega-Ruiz y Marín-López fue a partir de una media aritmética de las prevalencias (ya que realizaron una revisión sistemática, y no un meta-análisis), a diferencia de éste trabajo que pondera la estimación por la variabilidad del estudio; siendo ésta ultima la técnica más apropiada en la síntesis de resultados (Borenstein et al., 2009). Este efecto presente pero contrario en ambas investigaciones, señala la necesidad de estudiar el efecto de la cantidad de ítems en la estimación de la prevalencia del acoso escolar como señalan las autoras.

En conclusión, los resultados reportados tienen implicaciones importantes para el estudio y atención del bullying y cualquier forma de violencia que se de en las escuelas. Por una parte, es fundamental que los estudios se provean de información suficiente para estimar la prevalencia, pues no todos los estudios realizados fueron susceptibles de incluirse en este meta-análisis. Una recomendación es que los investigadores brinden tamaños de muestra y porcentaje de alumnos que sufren o realizan acoso escolar como parte de la descripción de la muestra de manera rutinaria. Así como también, realizar estudios experimentales para explorar el efecto de 
la cantidad de ítems en la estimación de prevalencias. Por otra parte, también es importante detallar adecuadamente el tipo de instrumentos utilizados en los futuros reportes (p. ej.: mencionar si se utilizó una definición y cuál fue en su caso; así como la forma de operacionalizar el concepto en caso de no usar una definición; y el número de reactivos para evaluarlo). Esto último es especialmente importante a la luz de que no todos los instrumentos utilizados evalúan los tres componentes esenciales del bullying, por lo que para propósitos de síntesis y desarrollo metodológico reportar estas características de los instrumentos puede ser un gran paso para mejorar los estudios del acoso escolar en México.

\section{Referencias}

Las referencias marcadas con un asterisco $\left({ }^{*}\right)$ fueron incluidas en el meta-análisis.

*Albores-Gallo, L., Sauceda-García, J. M., RuizVelasco, S., \& Roque-Santiago, E. (2011). El acoso escolar (bullying) y su asociación con trastornos psiquiátricos en una muestra de escolares en México. Salud Publica de Mexico, 53(3), 220-227.

*Alcántar Nieblas, C., Tánori Quintana, J., Valdés Cuervo, Á. A., \& Reyes Rodríguez, A. C. (2016). Manejo de la vergüenza de estudiantes de bachillerato con y sin reportes de ciberbullying hacia los pares. En J. A. Vera Noriega \& Á. A. Valdés Cuervo (Eds.), La violencia escolar en México. Temáticas y perspectivas de abordaje (pp. 41-50). Hermosillo: Centro de Investigación en Alimentación y Desarrollo, A.C.

*Avendaño Coronel, S. \& Figueroa Campos, M. (2013). Un estudio del acoso cibernético "cyberbullying" en estudiantes de la ENP y CCH de la UNAM: Incidencia y tipos, perfiles de agresores y víctimas y acciones de afrontamiento. En XII Congreso Nacional De Investigación Educativa (pp. 1-11).

*Avilés-Dorantes, D. S., Zonana-Nacach, A., \& Anzaldo-Campos, M. C. (2012). Prevalencia de acoso escolar (bullying) en estudiantes de una secundaria pública. Salud Publica de Mexico,
54(4), 362-363. Doi: https://doi.org/10.1590/ S0036-36342012000400002

Avilés Martínes, J. M. (1999). CIMEl. Cuestionario sobre intimidación y maltrato entre iguales. Valladolid: Autor.

Barendregt, J. J., Doi, S. A., Lee, Y. Y., Norman, R. E., \& Vos, T. (2013). Meta-analysis of prevalence. Journal of Epidemiology and Community Health, 67(11), 974-978. Doi: https://doi. org $/ 10.1136 /$ jech-2013-203104

Bauman, S., Toomey, R. B., \& Walker, J. L. (2013). Associations among bullying, cyberbullying, and suicide in high school students. Journal of Adolescence, 36(2), 341-350. Doi: https://doi. org/10.1016/j.adolescence.2012.12.001

*Bautista Hernández, G., Valdés Cuervo, A. A., \& Vera Noriega, J. A. (2013). Diferencias en el autoconcepto y clima familiar de subgrupos de estudiantes víctimas del bullying: víctimas y víctimas-agresores. En XII Congreso Nacional De Investigación Educativa (pp. 1-10). Guanajuato.

Borenstein, M., Hedges, L. V., Higgins, J. P. T., \& Rothstein, H. R. (2009). Introduction to MetaAnalysis. Sussex: John Wiley \& Sons. Doi: https://doi.org/10.1002/9780470743386

*Castillo Rocha, C. (2011). Juicio moral en conductas de maltrato entre iguales en una escuela secundaria de Mérida, Yucatán. En XI Congreso Nacional de Investigación Educativa (pp. 1-9). Ciudad de México.

Cerezo, F. (2000). El Test Bull-S. Instrumento para la evaluación de la agresividad entre escolares (Madrid). Madrid: Albor-Cohs.

Crothers, L. M. \& Levinson, E. M. (2004). Assessment of Bullying: A Review of Methods and Instruments. Journal of Counseling \& Development, 82(4), 496-503. Doi: https://doi. org/10.1002/j.1556-6678.2004.tb00338.x

Defensor del Pueblo. (2007). Violencia escolar: el maltrato entre iguales en la educación 
secundaria obligatoria 1999-2006. Nuevo estudio y actualización del Informe 2000. Madrid.

Del Rey, R. \& Ortega, R. (2005). Violencia interpersonal y gestión de la disciplina: Un estudio preliminar. Revista Mexicana de Investigación Educativa, 10(26), 805-832.

Dibomicucci Brazil, M., Basso Meneghini, V., de Souza Costa, P., \& Lemes, S. (2015). School Bullying Profile: a Bibliometric Study From 2000 To 2013. International Research Journal of Education and Innovation (IRJEI), 1(March), 120-131.

Felix, E. D., Sharkey, J. D., Green, J. G., Furlong, M. J., \& Tanigawa, D. (2011). Getting precise and pragmatic about the assessment of bullying: The development of the California Bullying Victimization Scale. Aggressive Behavior, 37(3), 234-247. Doi: https://doi.org/10.1002/ab.20389

Furlong, M. J., Sharkey, J. D., Felix, E. D., Tanigawa, D., \& Green, J. G. (2010). Bullying Assessment: A call for increased precision of self-reporting procedures. En S. R. Jimerson, S. M. Swearer, \& D. L. Espelage (Eds.), Handbook of Bullying in Schools: An International Perspective (pp. 329-346). New York: Routledge.

*García-Maldonado, G., Martínez-Salazar, G. J., Saldívar-González, A. H., Sánchez-Nuncio, R., Martínez-Perales, G. M., \& Barrientos-Gómez, M. del C. (2012). Factores de riesgo y consecuencias del cyberbullying en un grupo de adolescentes. Asociación con bullying tradicional. Boletín Medico del Hospital Infantil de Mexico, 69(6), 463-474.

Gini, G. \& Pozzoli, T. (2009). Association between bullying and psychosomatic problems: a metaanalysis. Pediatrics, 123(3), 1059-1065. Doi: https://doi.org/10.1542/peds.2008-1215

Gradinger, P., Strohmeier, D., \& Spiel, C. (2010). Definition and Measurement of Cyberbullying. Cyberpsychology: Journal of Psychosocial Research on Cyberspace, 4(2). Recuperado a partir de https://cyberpsychology.eu/article/ view/4235/3280
Green, J. G., Felix, E. D., Sharkey, J. D., Furlong, M. J., \& Kras, J. E. (2013). Identifying bully victims: definitional versus behavioral approaches. Psychological assessment, 25(2), 651-7. Doi: https://doi.org/10.1037/a0031248

*Joffre-Velázquez, V. M., García-Maldonado, G., Saldívar-González, A. H., Martínez-Perales, G., Lin-Ochoa, D., Quintanar-Martínez, S., \& Villasana-Guerra, A. (2011). Bullying en alumnos de secundaria. Características generales y factores asociados al riesgo. Boletín médico del Hospital Infantil de México, 68(3), 193-202.

*Lucio López, L. A. (2009). El Cyberbullying en estudiantes del nivel medio superior en México. $X$ Congreso Nacional de Investigación Educativa, (1998), 1-11.

*Madrid López, E. J., Valdés Cuervo, A. A., \& Vera Noriega, J. A. (2015). Diferencias en los estilos de afrontamiento de estudiantes víctimas y no involucrados en el bullying. En XIII Congreso Nacional de Investigación Educativa (pp. 1-9). Chihuahua.

Miranda, D. A., Serrano, J. M., Morales, T., \& Delgado, M. E. (2012). Diagnóstico de la agresión e intimidación en los planteles de la Escuela Preparatoria de la UAEMéx. México: Universidad Autónoma del Estadol de México.

Modecki, K. L., Minchin, J., Harbaugh, A. G., Guerra, N. G., \& Runions, K. C. (2014). Bullying prevalence across contexts: A meta-analysis measuring cyber and traditional bullying. Journal of Adolescent Health, 55(5), 602-611. Doi: https:// doi.org/10.1016/j.jadohealth.2014.06.007

*Murrieta, P., Ruvalcaba, N. A., Caballo, V. E., \& Lorenzo, M. (2014). Cambios en la percepción de la violencia y el comportamiento agresivo entre niños a partir de un programa de habilidades socioemocionales. Psicología Conductual: Revista Internacional Clínica y de la Salud, 22(3), 569-584.

Orpinas, P. (2009). Manual MeasurementAggression, Victimization, \& Social Skills Scales. Georgia: The University of Georgia. 
Ortega, R., Mora, J., \& Mora-Merchán, J. A. (1995). Cuestionario sobre intimidación y maltrato entre iguales. Sevilla.

*Prieto-Quezada, M. T., Carrillo Navarro, J. C., \& Lucio López, L. A. (2015). Violencia virtual y acoso escolar entre estudiantes universitarios: el lado oscuro de las redes sociales. Innovación Educativa, 15(68), 33-47. Doi: https://doi. org/10.11144/Javerianacali.PPSI14-1.rbcp

R Core Team. (2017). R: A Language and Environment for Statistical Computing. Vienna, Austria: R Foundation for Statistical Computing. Recuperado a partir de https:// www.r-project.org/

*Ramírez Almaraz, M., Díaz, F., Arceo, B., \& Flores, R. L. (2015). Maltrato entre escolares: Diagnóstico en estudiantes de nivel secundaria en la Ciudad de México. Psychology, Society, \& Education, 7(2), 169-182. Doi: https://doi. org/10.25115/psye.v7i2.531

*Ramos Herrera, M. A. \& Vázquez Valls, R. (2011). Bullying en el nivel superior. En XI Congreso Nacional de Investigación Educativa (pp. 1-6). Ciudad de México.

*Ruíz-Ramírez, R., Zapata-Martelo, E., García-Cué, J. L., Pérez-Olvera, A., Martínez-Corona, B., \& Rojo-Martínez, G. (2016). Bullying en una universidad agrícola del Estado de México. $R a$ Ximhai, 12(1), 105-126.

*Ruiz Armenta, M. B. (2015). Manifestaciones del cyberbullying a través de la red social Facebook y la telefonía móvil. En XIII Congreso Nacional de Investigación Educativa (pp. 1-14). Chihuahua.

Schwarzer, G. (2007). meta: An R package for metaanalysis. $R$ News, 7(3), 40-45.

Swearer, S. M., Siebecker, A. B., Johnsen-Frerichs, L. A., \& Wang, C. (2010). Assessment of Bullying/ Victimization: The problem of comparability across studies and across methodologies. En S. R. Jimerson, S. M. Swearer, \& D. L. Espelage (Eds.), Handbook of Bullying in Schools: An
International Perspective (pp. 305-328). New York: Routledge.

The Joanna Briggs Institute. (2014). The systematic review of prevalence and incidence data. The Joanna Briggs Institute Reviewers' Manual 2014. Adelaide: The Joanna Briggs Institute.

Valadez, B. (2014, mayo 23). México es el primer lugar de bullying a escala internacional. Milenio. com. Recuperado a partir de http://www.milenio.com/politica/Mexico-primer-bullyingescala-internacional_0_304169593.html

*Valadéz Figueroa, I., Amezcua Fernández, R., \& González Gallegos, N. (2009). Maltrato entre iguales psicopatología e intento suicida. En $X$ Congreso Nacional de Investigación Educativa (pp. 1-15). Veracruz.

*Valadez Figueroa, I. de la A., González Gallegos, N., Orozco Valerio, M. de J., \& Montes Barajas, R. (2011). Atribuciones causales del maltrato entre iguales: la perspectiva de los alumnos y del personal de escuelas de enseñanza media básica. Revista Mexicana de Investigación Educativa, 16(51), 1111-1136.

Valdés, A., Urías, M., Tapia, C., Torres, G., Carlos, E., Vera, J., \& Ponce, D. (2012). Caracterización de la violencia escolar en escuelas secundarias del Sur de Sonora (InformeTécnico). Sonora.

*Valdés Cuervo, Á. A., Alcántar Nieblas, C., Reyes Rodríguez, A. C., Torres Acuña, G. M., \& Urías Murrieta, M. (2014). Diferencias en el autoconcepto de estudiantes de bachillerato con y sin reportes de victimización por ciberbullying. $L a$ Sociedad Académica, 22(43), 35-39.

Valdés Cuervo, Á. A., Alcántar Nieblas, C., Tánori Quintana, J., \& Torres Acuña, G. M. (2016). Apuntes y análisis de la investigación acerca de la violencia entre estudiantes en México. En R. García Flores, S. V. Mortis Lozoya, J. Tánori Quintana, \& T. I. Sotelo Quiñonez (Eds.), Educación y Salud: Evidencias y propuestas de investigación en Sonora (pp. 70-78). Sonora: Instituto Tecnológico de Sonora. 
*Valdés Cuervo, Á. A. \& Carlos Martínez, E. A. (2014). Relación entre el autoconcepto social, el clima familiar y el clima escolar con el bullying en estudiantes de secundarias. Avances en psicología latinoamericana, 32(3), 447-457. Doi: https://doi.org/10.12804/apl32.03.2014.07

*Valdés Cuervo, Á. A., Carlos Martinez, E. A., Tanori Quintana, J., \& Wendlandt Amezaga, T. R. (2014). Differences in Types and Technological Means by Which Mexican High Schools Students Perform Cyberbullying: Its Relationship with Traditional Bullying. Journal of Educational and Developmental Psychology, 4(1), 105-113. Doi: https://doi.org/10.5539/jedp.v4n1p105

Valdés Cuervo, Á. A., Carlos Martínez, E. A., \& Torres Acuña, G. M. (2012). Diferencias en la situación socioeconómica, clima y ajuste familiar de estudiantes con reportes de bullying y sin ellos. Psicología desde el Caribe, 29(3), 616-631.

Valdés Cuervo, Á. A., Ojeda García, A., Urías Murrieta, M., \& Ponce Segura, D. B. (2013). Factores de riesgo en estudiantes de secundaria con conductas violentas hacia sus compañeros. En J. J. Vales García, Á. A. Valdés Cuervo, J. Angulo Armenta, C. García Hernández, \& I. González Castro (Eds.), Investigación psicoeducativa en Sonora: Resultados y propuestas de acción (pp. 1-19). Ciudad de México: Pearson.

Valdés Cuervo, Á. A., Sanchez Escobedo, P. A., \& Carlos Martínez, E. A. (2012). Autoconcepto social y ajuste escolar de estudiantes de educación media con conductas de hostigamiento en la escuela. Revista "Educación y Ciencia", 2(40), 85-96.

*Valdés Cuervo, Á. A., Yañez Quijada, A. I., \& Carlos Martínez, E. A. (2013). Diferencias entre subgrupos de estudiantes involucrados en el bullying: víctimas, agresores-víctimas y agresores. Liberabit, 19(2), 1729-4827.

*Vega López, M. G. \& González Pérez, G. J. (2016). Bullying en la escuela secundaria: Factores que disuaden o refuerzan el comportamiento agresor de los adolescentes. Revista Mexicana de Investigacion Educativa, 21(71), 1165-1189.
*Vega López, M. G., González Pérez, G. J., \& Quintero Vega, P. P. (2013). Ciberacoso: victimización de alumnos en escuelas secundarias públicas de Tlaquepaque, Jalisco, México. Revista de Educación y Desarrollo, 25(2), 13-20.

*Vega López, M. G., González Pérez, G. J., Valle Barbosa, M. A., Flores Villavicencio, M. E., \& Vega López, A. (2013). Acoso escolar en la zona metropolitana de Guadalajara, México: prevalencia y factores asociados. Salud Colectiva. Universidad Nacional de Lanús, 9(2), 183-194. Doi: https://doi.org/10.18294/sc.2013.31

*Velázquez Reyes, L. M. (2011). Sexting, Sexcasting, Sextorsión, Grooming y Cyberbullyying. El lado oscuro de las TICs. XI Congreso Nacional de Investigación Educativa, 1-9.

*Vilchis, R. M., Rivera, J. P., Arriga, K. J., Reynoso, T. M., Miranda, D. A., Maya, M. E. D., ... Cuenca Sánchez, V. (2015). Prevención de la violencia escolar cara a cara y virtual en bachillerato. Psychology, Society and Education, 7(2), 201212. Doi: https://doi.org/10.25115/psye.v7i2.533

Vivolo-Kantor, A. M., Martell, B. N., Holland, K. M., \& Westby, R. (2014). A systematic review and content analysis of bullying and cyber-bullying measurement strategies. Aggression and Violent Behavior, 19(4), 423-434. Doi: https:// doi.org/10.1016/j.avb.2014.06.008

Volk, A. A., Dane, A. V., \& Marini, Z. A. (2014). What is bullying? A theoretical redefinition. Developmental Review, 34(4), 327-343. Doi: https://doi.org/10.1016/j.dr.2014.09.001

Volk, A. A., Veenstra, R., \& Espelage, D. L. (2017). So you want to study bullying? Recommendations to enhance the validity, transparency, and compatibility of bullying research. Aggression and Violent Behavior, 36(July), 34-43. Doi: https://doi.org/10.1016/j.avb.2017.07.003

Ybarra, M. L., Espelage, D. L., \& Mitchell, K. J. (2014). Differentiating youth who are bullied from other victims of peer-aggression: The importance of differential power and repetition. Journal of Adolescent Health, 
55(2), 293-300. Doi: https://doi.org/10.1016/j. jadohealth.2014.02.009

Zych, I., Ortega-Ruiz, R., \& Marín-López, I. (2016). Cyberbullying: a systematic review of research, its prevalence and assessment issues in Spanish studies. Psicología Educativa, 22(1), 5-18. Doi: https://doi.org/10.1016/j.pse.2016.03.002 